\title{
Efecto del conflicto decisional sobre el control del asma en escolares de 10 a 14 años
}

\author{
DANIEL CIUDAD A.*,**,***, GABRIELA BRAVO S.*,***, \\ LINRAYÉN CONTRERAS A.*,*** Y YOCELIN HUENUL V.*,***
}

\section{Effect of decisional conflict on the control of asthma in scholars from 10 to 14 years old}

Introduction: The number of asthmatic patients attending the public health system is increasing. However, the rate of adherence to treatment is very low. Adolescents have the largest percentage of abandonment to treatment, lack of control and a great decisional conflict (DC). The objective of this stu$d y$ was to evaluate the effect of the application of counseling on the DC in relation to asthma treatment and the level of control of their disease. Materials and Methods: Pre-experimental study that recruited 32 asthmatic children from 10 to 14 years-old, from the children's respiratory outpatients clinic of Carlos Van Buren hospital in Valparaiso, Chile. Only 15 children were willing to participate in the study and completed all of the counseling sessions. To determine the degree of DC of its pathology, the Ottawa Decision Conflict Scale was applied; and for the Asthma Control level, the Global Initiative for Asthma Scale (GINA) was used. Results: Mean children age was $12.06 \pm 1.16$ years-old. After the intervention, the level of Asthma Control was maintained and the mean of the DC decrease from 34.05 \pm 4.59 to $18.02 \pm 3.01$ points $(p<0.05$; paired Student's $t$-test). $73.3 \%$ of the patients lowered their level of decisional conflict. Conclusion: The counseling of decision support proved to have positive effects on the population studied.

Key words: Asthma; Adolescent; Counseling; Outpatients; Decisional Health Conflict.

\section{Resumen}

Introducción: La cantidad de pacientes asmáticos que asisten al sistema público de salud es cada vez mayor, no obstante, la tasa de adherencia al tratamiento es muy baja, siendo los adolescentes quienes presentan mayor porcentaje de abandono al tratamiento, inasistencia a sus controles y gran conflicto decisional (CD). El objetivo de este estudio fue evaluar el efecto de la aplicación de consejerías sobre el CD en relación al tratamiento del asma y el nivel de control de su enfermedad. Materiales y Métodos: Estudio pre-experimental que reclutó a 32 niños asmáticos entre 10 a 14 años de edad del policlínico respiratorio infantil del hospital Carlos Van Buren de Valparaíso. Solo 15 niños estuvieron dispuestos a participar en el estudio quienes completaron la totalidad de las sesiones de consejería. Para determinar el grado de CD de su patología, se aplicó la Escala de Conflicto Decisional de Ottawa; y para el nivel del control del asma, se usó la Escala Global Initiative for Asthma (GINA). Resultados: La edad media del grupo de niños fue de 12,06 \pm 1,16 años. Finalizada la intervención, el nivel de control de asma se mantuvo y la media del CD disminuyó de 34,05 $\pm 4,59$ a 18,02 $\pm 3,01$ puntos ( $p<0,05$; $t$ de Student para muestras pareadas). Un 73,3\% de los pacientes disminuyó su nivel de conflicto decisional. Conclusión: Las consejerías de apoyo decisional demostraron tener efectos positivos en la población estudiada.

Palabras clave: Asma; Adolescente; Consejería; pacientes ambulatorios; Conflicto Decisional en Salud.

\footnotetext{
* Escuela de Kinesiología, Universidad de Valparaíso. Valparaíso, Chile.

** Policlínico Respiratorio Infantil, Hospital Carlos Van Buren. Valparaíso, Chile.

*** Kinesiólogo.
} 


\section{Introducción}

La cantidad de pacientes asmáticos que acuden al sistema público de salud es cada vez mayor, pero del total de la población, son muy pocos los que asisten a todos sus controles, siendo los adolescentes quienes presentan mayor tasa de abandono al tratamiento ${ }^{1}$. A nivel público, las estrategias apuntan a dar todas las condiciones necesarias para que cada usuario asista a sus controles y continúe su tratamiento. Sin embargo, se deja de lado el rol de la educación y el conocimiento de su patología como parte fundamental de la intervención ${ }^{2-4}$.

Por otro lado, en el ámbito sanitario, tomar decisiones es una situación frecuente, aunque potencialmente difícil, provocando un conflicto decisional (CD) en las personas ${ }^{5-7}$. El CD corresponde a la incertidumbre personal acerca de qué curso de acción tomar, entre opciones competitivas que puedan implicar riesgo, pérdida, pesar o desafío a los propios valores 8 .

En la actualidad, si bien existe consensos participativos e integrales en relación al manejo del asma infantil, generalmente son los médicos quienes deciden por el paciente en función de lo que es mejor para su salud; agregando a lo anterior una población de adolescentes, quienes aún están bajo la supervisión de sus padres o tutores, tendremos una población con baja adherencia, escaso conocimiento de la patología y poco in- terés en el control de la misma9. El objetivo del presente estudio fue aplicar consejerías de apoyo decisional, basado en el modelo de toma de decisiones en salud de Ottawa ${ }^{10}$, en niños asmáticos y evaluar su efecto sobre el conflicto decisional en relación al tratamiento del asma y el nivel de control de su enfermedad.

\section{Materiales y Métodos}

Se presenta un estudio pre-experimental, no probabilístico, con pre y post test, sin grupo control que reclutó a 32 niños con diagnóstico de asma bronquial, en cualquier de sus tres niveles. Los participantes eran niños entre 10 a 14 años, asistentes al policlínico respiratorio infantil, del hospital Carlos Van Buren de Valparaíso, durante los años 2016 y 2017. Dentro de los criterios de exclusión se consideró aquellos adolescentes sin la confirmación médica de asma, fuera del rango etario, que decidieron seguir su tratamiento en cualquier otro centro de atención de salud, menores con problemas para entender instrucciones y que no hubieran firmado el asentimiento informado, como así sus padres el documento de consentimiento informado (Figura 1).

Cada uno de los sujetos de estudio participó de cuatro sesiones de consejerías con distintos temas de interés en relación a su enfermedad: a) ¿qué es el asma? b) farmacología y técnica inhalatoria; c)

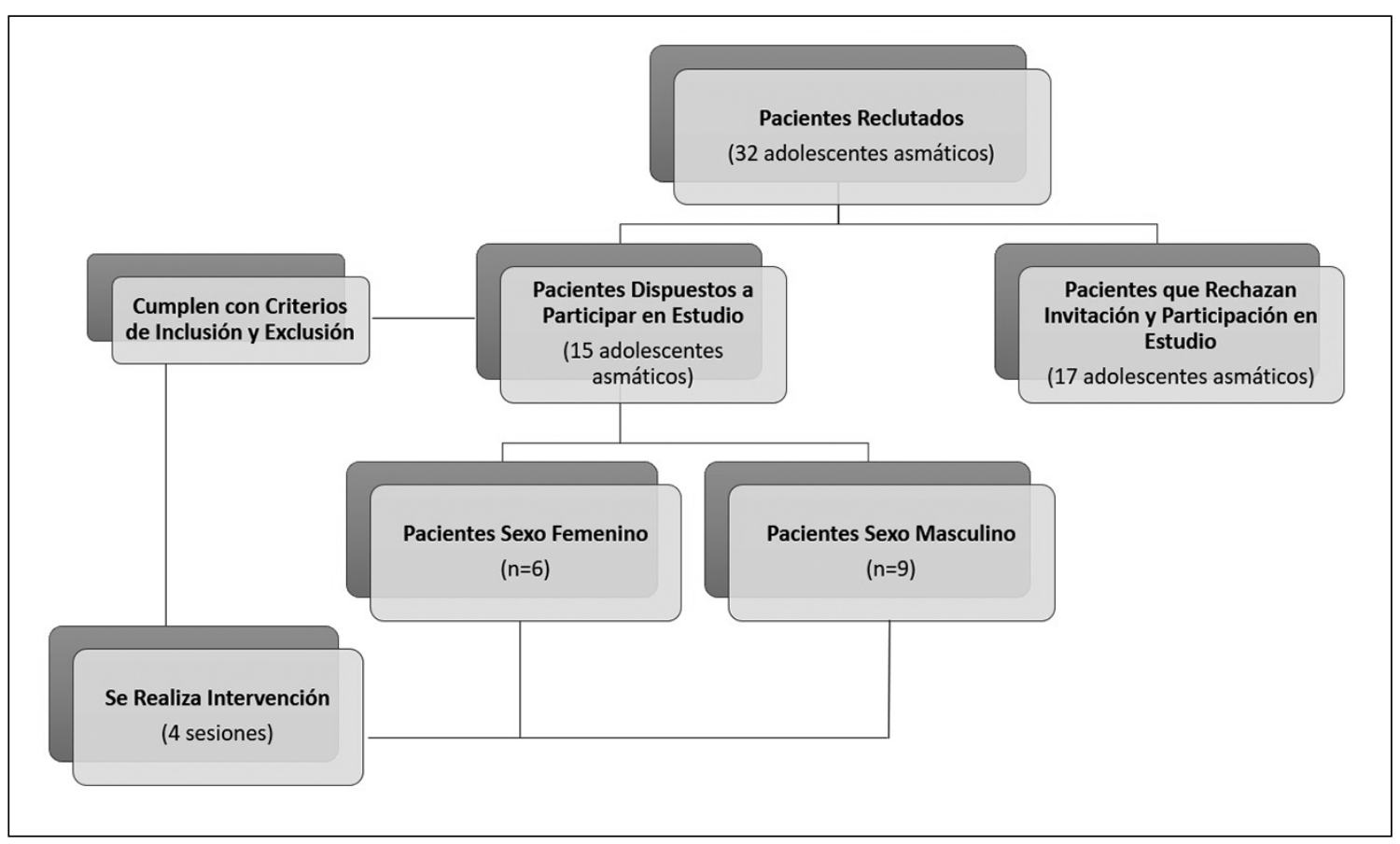

Figura 1. Flujograma del reclutamiento de pacientes participantes en el estudio. 
control de factores ambientales; d) educación al paciente en integración familiar. Las sesiones se realizaron cada 2 semanas, teniendo una duración máxima de $45 \mathrm{~min}$, los menores podían estar acompañados o no de sus familiares y la dinámica de enseñanza contempló trabajos grupales e individuales con material audiovisual, modelos 3D del sistema respiratorio, muestra de medicamentos y diferentes dispositivos de entrega de fármacos inhalados como de espaciadores y aero-cámaras. Cartillas informativas, fichas resumen de sesión y juegos interactivos en relación al control del asma. Las reuniones se llevaron a cabo después del horario de clases, en la jornada de la tarde, en las dependencias de la Universidad de Valparaíso.

Antes y después de la intervención a los niños se les aplicó, en forma individual y sin la pre- sencia de sus familiares o tutores, la 'Escala de Conflicto Decisional' (ECD) de Ottawa para determinar el grado del CD de su patología; y para establecer el nivel de control de su enfermedad la 'Escala Global Initiative for Asthma' (GINA). La escala GINA (2017) divide en tres los niveles de control del asma según la sintomatología y uso de medicación que presenta el sujeto: totalmente controlado, parcialmente controlado y no controlado (Tabla 1). ECD es una escala de tipo Likert de 16 ítems, los cuales son distribuidos en 5 subescalas: conocimiento e información, claridad de valores personales, apoyo percibido, incertidumbre y decisión efectiva (Tabla 2). Para obtener los resultados, se realiza una valoración de los puntajes de cada una de las 5 subescalas (0: totalmente de acuerdo; 1 : de acuerdo; 2 : indiferente; 3: desacuerdo; 4: totalmente en desacuer-

Tabla 1. Clasificación del asma según niveles de control*

\begin{tabular}{|c|c|c|c|c|}
\hline \multicolumn{5}{|c|}{ Nivel de control de los síntomas } \\
\hline $\begin{array}{l}\text { En las últimas } 4 \text { semanas el paciente ha } \\
\text { presentado }\end{array}$ & & $\begin{array}{l}\text { Bien } \\
\text { controlados }\end{array}$ & $\begin{array}{l}\text { Parcialmente } \\
\text { controlados }\end{array}$ & $\begin{array}{l}\text { Mal } \\
\text { controlados }\end{array}$ \\
\hline Síntomas diurnos más de 2 veces/semana & Sí $\square$ No $\square$ & \multirow{4}{*}{$\begin{array}{l}\text { Ninguno de } \\
\text { ellos }\end{array}$} & \multirow{4}{*}{$1-2$ de ellos } & \multirow{4}{*}{ 3-4 de ellos } \\
\hline Algún despertar nocturno por el asma & Sí $\square$ No $\square$ & & & \\
\hline $\begin{array}{l}\text { Necesidad de utilizar medicación sintomática* } \\
\text { más de dos veces/semana }\end{array}$ & Sí $\square$ No $\square$ & & & \\
\hline Alguna limitación de la actividad debida al asma & Sí $\square$ No $\square$ & & & \\
\hline
\end{tabular}

*GINA 2017.

Tabla 2. Escala de Conflicto Decisional

\begin{tabular}{|c|c|}
\hline Subescalas & Preguntas \\
\hline Incertidumbre & $\begin{array}{l}\text { 1. Esta es una decisión fácil de tomar para mí } \\
\text { 2. Yo estoy seguro de lo que tengo que hacer en esta decisión } \\
\text { 3. Está claro cuál es la mejor opción para mí }\end{array}$ \\
\hline $\begin{array}{l}\text { Conocimiento e } \\
\text { información }\end{array}$ & $\begin{array}{l}\text { 4. Yo estoy consciente de las diferentes opciones que tengo en esta decisión } \\
\text { 5. Yo siento que conozco los aspectos positivos de cada opción } \\
\text { 6. Yo siento que conozco los aspectos negativos de cada opción }\end{array}$ \\
\hline $\begin{array}{l}\text { Claridad valores } \\
\text { personales }\end{array}$ & $\begin{array}{l}\text { 7. Yo tengo claridad acerca de cuán importantes son los aspectos positivos para mí } \\
\text { 8. Yo tengo claridad acerca de cuán importantes son los aspectos negativos para mí } \\
\text { 9. Yo tengo claridad acerca de qué es lo más importante para mí (los aspectos positivos } \\
\text { o los aspectos negativos) }\end{array}$ \\
\hline Ароуо & $\begin{array}{l}\text { 10. Yo estoy tomando esta decisión sin ninguna presión de otras personas } \\
\text { 11. Yo tengo la cantidad apropiada de apoyo de otros para tomar esta decisión } \\
\text { 12. Yo cuento con suficientes consejos acerca de las opciones }\end{array}$ \\
\hline Decisión efectiva & $\begin{array}{l}\text { 13. Yo siento que he hecho una elección bien informada } \\
\text { 14. Mi decisión muestra lo que es importante para mí } \\
\text { 15. Yo espero mantenerme firme en mi decisión } \\
\text { 16. Yo estoy satisfecho/a con mi decisión }\end{array}$ \\
\hline
\end{tabular}

Tabla de subescalas Conflicto Decisional. Original de A. O’Connor, 1993; Revisada en marzo de 1997; Versión 1998 Traducción Mila Urrutia, 1999. 
do). El puntaje total se divide por 16 y luego se multiplica por 25; el menor puntaje es cero que significa la nula existencia de CD y un máximo de 100 que corresponde a un alto índice de CD. El puntaje de corte utilizado para determinar CD es igual a 25 puntos $^{7}$.

\section{Aspectos éticos}

Este estudio fue aprobado por el Comité de Bioética de la Facultad de Medicina de la Universidad de Valparaíso, código 017/2016.

\section{Análisis estadístico}

Para obtener los resultados estadísticos se utilizó el Software Graphpad InStat 3.0. Se compararon los diferentes ítems de la ECD y se analizó el nivel de control del asma. La distribución normal de la variable se determinó con la prueba de Shapiro-Wilks, estableciéndose un nivel de significancia del 0,05. Para comparar los valores obtenidos pre y post intervención se aplicó la prueba $t$ de Student para muestras relacionadas.

\section{Resultados}

La muestra correspondió a 15 niños, 9 hombres y 6 mujeres con una edad promedio de 12,06 $\pm 1,16$ años. El nivel de control del asma, previo a la intervención, fue de 14 niños en condición de asma controlada y un niño con asma parcialmente controlada. Posterior a la intervención no se observaron cambios.

En cuanto al nivel de CD presentado por los participantes, se obtuvo una media de $34,05 \pm$ 4,59 puntos, antes de las consejerías de educación y posterior a la intervención una media de 18,02 $\pm 3,01$ puntos. El detalle de la puntuación de cada dimensión en la ECD, antes y después de la intervención, se muestra en la Tabla 3. La Tabla 4 resume los valores estadísticos para el ítem con-

Tabla 3. Puntuación de Escala Conflicto Decisional

\begin{tabular}{|c|c|c|c|c|c|c|c|c|c|c|c|c|}
\hline \multirow[t]{3}{*}{ Sujetos } & \multicolumn{10}{|c|}{ Puntuación por Subescalas } & \multicolumn{2}{|c|}{ Puntaje total } \\
\hline & \multicolumn{2}{|c|}{ Informado } & \multicolumn{2}{|c|}{ Valores } & \multicolumn{2}{|c|}{ Apoyo } & \multicolumn{2}{|c|}{ Incertidumbre } & \multicolumn{2}{|c|}{ Decisión } & \multirow[b]{2}{*}{$\mathbf{A}$} & \multirow[b]{2}{*}{ D } \\
\hline & $\mathbf{A}$ & D & $\mathbf{A}$ & D & $\mathbf{A}$ & D & A & D & $\mathbf{A}$ & D & & \\
\hline $1 \mathrm{M}$ & 0 & 66,67 & 33,33 & 0 & 66,67 & 0 & 33,33 & 0 & 0 & 0 & 25 & 12,5 \\
\hline $2 \mathrm{M}$ & 100 & 66,67 & 0 & 33,33 & 33,33 & 66,67 & 33,33 & 33,33 & 100 & 0 & 56,25 & 37,5 \\
\hline $3 \mathrm{H}$ & 100 & 33,33 & 66,67 & 0 & 0 & 0 & 66,67 & 0 & 25 & 6,25 & 50 & 7,81 \\
\hline $4 \mathrm{M}$ & 8,33 & 33,33 & 25 & 58,33 & 16,67 & 33,33 & 16,67 & 25 & 0 & 25 & 12,5 & 34,38 \\
\hline $5 \mathrm{H}$ & 33,33 & 41,67 & 33,33 & 25 & 0 & 25 & 16,67 & 33,33 & 12,5 & 25 & 18,75 & 29,69 \\
\hline $6 \mathrm{H}$ & 25 & 0 & 8,33 & 33,33 & 8,33 & 25 & 0 & 0 & 6,25 & 0 & 9,38 & 10,94 \\
\hline $7 \mathrm{M}$ & 66,67 & 8,33 & 66,67 & 0 & 33,33 & 0 & 33,33 & 8,33 & 0 & 0 & 37,5 & 3,13 \\
\hline $8 \mathrm{H}$ & 25 & 0 & 66.67 & 33,33 & 66,67 & 0 & 25 & 0 & 37,5 & 0 & 43,75 & 6,25 \\
\hline $9 \mathrm{H}$ & 33,33 & 33,33 & 33,33 & 0 & 33,33 & 25 & 33,33 & 8,33 & 0 & 0 & 25 & 12,5 \\
\hline $10 \mathrm{H}$ & 8,33 & 25 & 33,33 & 8,33 & 16,67 & 25 & 8,33 & 8,33 & 12,5 & 12,5 & 15,63 & 15,63 \\
\hline $11 \mathrm{M}$ & 8,33 & 66,67 & 66,67 & 0 & 16,67 & 0 & 33,33 & 33,33 & 87,5 & 25 & 45,31 & 25 \\
\hline $12 \mathrm{H}$ & 100 & 33,33 & 100 & 100 & 83,33 & 25 & 16,67 & 0 & 12,5 & 25 & 59,38 & 35,94 \\
\hline $13 \mathrm{H}$ & 0 & 0 & 33,33 & 33,33 & 8,33 & 0 & 33,33 & 0 & 6,25 & 0 & 15,63 & 6,25 \\
\hline $14 \mathrm{M}$ & 66,67 & 0 & 0 & 25 & 66,67 & 66,67 & 16,67 & 0 & 37,5 & 12,5 & 37,5 & 20,31 \\
\hline $15 \mathrm{H}$ & 8,33 & 33,33 & 25 & 0 & 83,33 & 0 & 66,67 & 25 & 100 & 6,25 & 59,38 & 12,5 \\
\hline
\end{tabular}

H: hombres; M: mujeres; A: antes; D: después. Puntajes iguales o mayores a 25 puntos determina CD.

Tabla 4. Valores estadísticos para el ítem Conflicto Decisional total

\begin{tabular}{|ccc|}
\hline Valores & Previo a la intervención & Posterior a la intervención \\
\hline $\mathrm{n}$ & 15 & 15 \\
Media & 34,05 & $18,02^{*}$ \\
\hline Error estándar & 4,59 & 3,01 \\
\hline
\end{tabular}

$* p<0,05$. Los resultados pasaron la prueba de normalidad de Shapiro-Wilks. Para comparar los valores obtenidos pre y postintervención se aplicó la prueba t de Student para muestras relacionadas. 


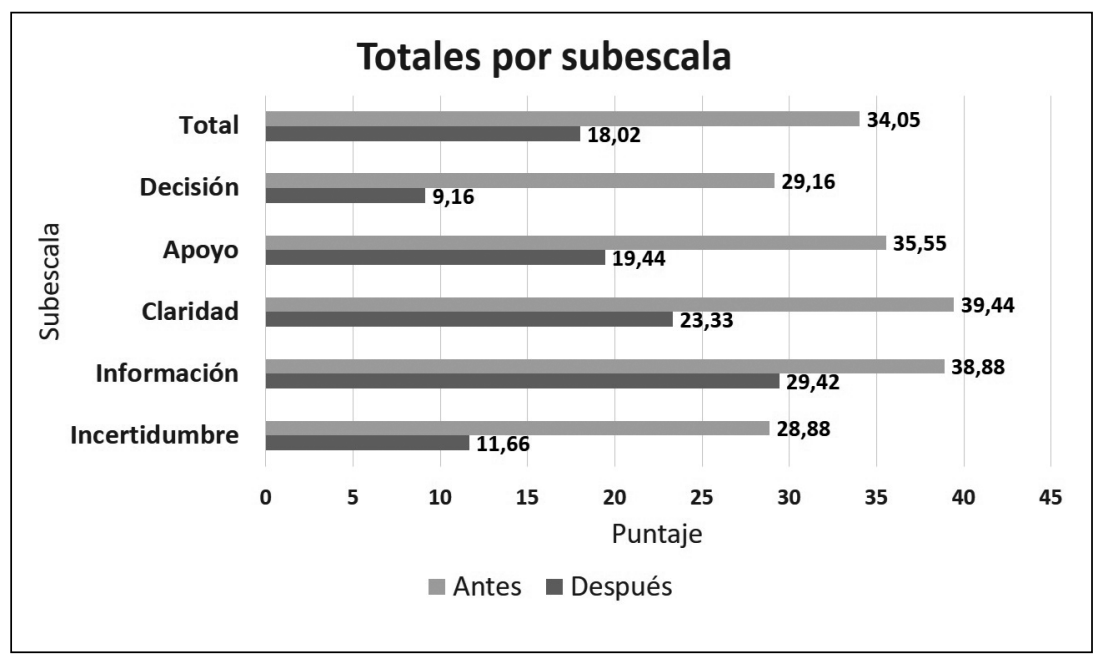

Figura 2. Puntuación Escala de Conflicto Decisional por subescalas. Con $\mathrm{p}<0,05$ para subescala incertidumbre y resultado total. Se aplicó prueba $\mathrm{t}$ de Student para muestras relacionadas para comparar los puntajes pre y post intervención. flicto decisional total y la Figura 2 los puntajes totales registrado en cada una de las subescalas.

Del total de los participantes un 73,3\% disminuyó su CD; un $6,7 \%$ se mantuvo igual y un $20 \%$ aumentó el nivel de conflicto decisional.

\section{Discusión}

Los resultados del presente estudio son similares a los reportados por otras investigaciones ${ }^{11,12}$, las cuales sugieren considerar las consejerías de educación como una herramienta beneficiosa en el proceso de toma de decisiones sobre el tratamiento, constituyendo a las consejerías como una alternativa de intervención en la población ${ }^{6}$.

$\mathrm{Al}$ analizar el comportamiento de los niños en la subescala "conocimiento e información", el $40 \%$ de los participantes, previo a la intervención, manifestaron conocer los aspectos positivos y negativos del tratamiento de su asma, además de conocer las diferentes opciones que presentaban; posterior a la intervención solo un 33,3\% manifestó sentirse informado respecto al tratamiento de su enfermedad. Por otro lado, si analizamos la subescala "incertidumbre", el 60\% de la muestra manifestó estar inseguro sobre cuál es la mejor elección a realizar con respecto a su patología, antes de la intervención. Lo anterior permite visualizar que la toma de decisión en salud para un niño/adolescente es compleja, debido a la existencia de componentes emocionales y cognitivos. La inmadurez del adolescente lo lleva a evadir responsabilidades, recurriendo generalmente a la opinión de los padres, otorgándoles poder directo $\mathrm{o}$ indirecto en la toma de decisión. Algunos estudios afirman que son los padres quienes poseen un papel crucial en la ayuda y apoyo psicológico de los niños y adolescentes, para alcanzar el control de su asma ${ }^{13,14}$, el cual coincide con un estudio de autoeficacia, apoyo social y calidad de vida realizado por Avendaño ${ }^{15}$. Una de las variables que más influye en la conducta de los padres de niños asmáticos y el tratamiento del asma, es la percepción que se tiene sobre esta, influenciada por el desconocimiento sobre la misma, provocando que la mayoría de los niños asuman conductas de riesgo, como la escasa adherencia al tratamiento y falta de interés en alcanzar el control de su enfermedad ${ }^{16}$.

Si observamos la subescala "decisión efectiva", antes de la intervención, el $60 \%$ de los encuestados, se siente satisfecho con la decisión tomada respecto al tratamiento de su enfermedad y el $20 \%$, en la subescala "claridad de valores", respondió sentirse claro sobre los valores asignados a cada una de las opciones. Después de realizar consejerías, un 73,3\% respondió sentirse satisfecho con la decisión tomada y un $46,6 \%$ manifiesta sentirse claro sobre los valores asignados a las opciones a elegir respecto al tratamiento de su asma, como también a los beneficios y riesgos asociados a dicha elección. Una revisión sistemática de la literatura al respecto, reveló que no existe un comportamiento típico, en el adolescente, para una deficiente adherencia a su tratamiento, factores como: creencias, autogestión, función del cuidador y educación en salud son importantes de trabajar al momento para mejorar el éxito de la adherencia al tratamiento ${ }^{17}$. Las consejerías durante la toma de decisiones otorgan a los participantes más herramientas para poder enfrentarse a su enfermedad, que eventualmente podrían mejorar su condición de salud logrando 
mantener el nivel de control, debido a una mayor adherencia al tratamiento escogido, más aún si se tiene en cuenta las preferencias, creencias y valores del mismo paciente ${ }^{18}$. Lo anterior cobra mayor sentido en este estudio en que la mayoría de los niños mantuvieron la condición de asma controlada (GINA), y en donde la consejería buscó empoderarlos en el control de su enfermedad, lo cual es el principal objetivo de la intervención.

Otro punto importante a considerar es el apoyo familiar que presentaron los menores durante el tratamiento de su enfermedad, el cual va a ser un punto fundamental en la expresión del control y por ende la disminución de crisis, ubicando a los pacientes en un estadio de controlados o parcialmente controlados. En el caso de los participantes de nuestro estudio, todos asistieron a las consejerías en compañía de al menos uno de sus padres o tutores, lo que demuestra el gran interés que presentan frente a la enfermedad de sus hijos. Por lo tanto, queda claro que son los padres quienes desempeñan un papel fundamental en la ayuda de los niños y adolescentes con asma, así como en el apoyo, autocontrol y conocimiento de la enfermedad ${ }^{19}$. Al respecto, el $60 \%$ de la muestra manifiesta sentir mayor apoyo, por parte de su familia, posterior de la intervención; más aún cuando ésta toma en cuenta las preferencias y los valores de los niños, la probabilidad de adhesión a la opción elegida de tratamiento aumenta considerablemente $6,13,19$.

Finalmente es de nuestro interés dar la debida importancia a la educación en el tratamiento del niño con asma bronquial, mostrando nuestra experiencia y buscando evidencia científica. Al respecto, debemos volver a mencionar que la presente investigación no considera la participación de un grupo control, por lo cual sus resultados se deben limitar a la muestra en estudio.

\section{Conclusión}

Los niveles de conflicto decisional respecto a la enfermedad disminuyeron de manera global en 11 de los 15 menores, demostrando un aporte significativo de las consejerías en su toma de decisión final. Si bien el nivel de control del asma en los menores se mantuvo sin variación, dicho resultado es considerado como beneficioso dadas las características de una patología como el Asma, donde lo que se persigue es su control, evitando las exacerbaciones.

La principal limitación de la presente investigación es la inexistencia de un grupo control de estudio, otorgando una menor validez a los resultados obtenidos.

\section{Bibliografía}

1.- VELÁSTEGUI C, PÉREZ-CANTO P, ZÁRATE V, ARENAS D, SALINAS P, MORENO G, et al. Impacto del asma en escolares de dos centros de salud primaria. Rev Med Chile 2010; 138: 205-12.

2.- HENRÍQUEZ M, CERUTI E. Educación en niños asmáticos controlados en hospital Roberto del Río. Impacto en conocimientos, adherencia y control de la enfermedad. Rev Chil Enferm Respir 2013; 29: 70-4.

3.- CIRIA A, CAPOTE A, SARDIÑAS S, GARCÍAA. Los procesos sociales y medioambientales en la salud de niños asmáticos: desafíos para la ciencia y la tecnología en Cuba. Rev Cubana Med Gen Integr 2016; 32: $93-$ 106.

4.- HERRERA A, LEÓN A, UBILLA C, PÉREZ M, LOZANO J. Utilidad de la educación en asma bronquial infantil: experiencia piloto. Rev Chil Enferm Respir 2014, 30: 197-202.

5.- PERESTELO PÉREZ L. Manual con criterios de evaluación y validación de las herramientas de ayuda para la toma de decisiones. Ministerio de Sanidad, Servicios Sociales e Igualdad. Servicio de evaluación del servicio Canario de la Salud; 2013.

6.- ANNICK DES CORMIERS A, LÉGARÉ F, SIMARD S, BOULET L. Decisional conflict in asthma patients: a cross sectional study. J Asthma 2015; 52:1084-91, DOI: 10.3109/02770903.2015.1047955.

7.- $\quad$ BRAVO P, DOIS A, HERNÁNDEZ M, VILLARROEL L. Conflicto decisional en personas con diabetes mellitus e hipertensión arterial usuarias del nivel primario de atención en salud de Chile. Rev Med Chile 2018; 146 : 1286-93.

8.- O'CONNOR A, STACEY D, BOLAND L. Ottawa decision support tutorial. Ottawa Hospital Research Institute. 2007-2015. Disponible en: https://decisionaid. ohri.ca/ODST/pdfs/ODST.pdf.

9.- HERRERA A, ABARA S, ÁLVAREZ C, ASTUDILLO C, CORRALES R, CHALA E, et al. Consenso chileno SER-SOCHINEP para el manejo del asma severo en el niño. Rev Chil Enferm Respir 2016; 32: 100-12.

10.- URRUTIA M, CAMPOS S, O'CONNOR A. Validación de una versión en español de la Escala de Conflicto Decisional. Rev Med Chile 2008; 136: 1439-47.

11.- LIPSTEIN EA, MUETHING KA, DODDS CM, BRITTO MT. "I'm the one taking it": adolescent participation in chronic disease treatment decisions. J Adolesc Health 2013; 53: 253-9.

12.- MOSQUEDA D. Efecto de una consejería de apoyo decisional en salud en adolescentes con malnutrición por exceso. 2016. (Disertación doctoral), Universidad de Concepción. Facultad de Enfermería (Campus Concepción).

13.- BENDER BG, ANNETT RD, IKLÉ D, DUHAMEL TR, RAND C, STRUNK RC. Relationship between disease and psychological adaptation in children in the Child- 
hood Asthma Management Program and their families. CAMP Research Group. Arch Pediatr Adolesc Med 2000; 154: 706-13.

14.- CHERNOFF RG, IREYS HT, DEVETKA, KIM YJ. A randomized, controlled trial of a community-based support program for families of children with chronic illness: pediatric outcomes. Arch Pediatr Adolesc Med 2002; 156: 533-9.

15.- AVENDAÑO M, BARRA E. Autoeficacia, Apoyo Social y Calidad de Vida en Adolescentes con Enfermedades Crónicas. Terapia psicológica 2008; 26 (2): 165-72. Disponible en: http://dx.doi.org/10.4067/S071848082008000200002.

16.- RAMÍREZ G, BARRERA L, RAMÍREZ Y, QUICENO A, AGUDELO A, HENAO D. Creencias familiares y adherencia al tratamiento en pacientes pediátricos con asma: estudio mixto, 2013-2014. Archivos de Medicina (Col) 2016; 16: 74-88 .

17.- AHMAD A, SORENSEN K. Enabling and hindering factors influencing adherence to asthma treatment among adolescents: A systematic literature review. Journal of Asthma 2016; 53: 862-78.

18.- DES CORMIERS A, LÉGARÉ F, SIMARD S, BOULET L. Decisional conflict in asthma patients: a cross sectional study. Journal of Asthma 2015; 52: 1084-91.

19.- FIGUEREDO M, FABRÉ D, COUTIN G, DEL ARCO A. Evaluación del impacto de un programa educativo en algunos factores emocionales de niños asmáticos y sus padres. Rev Alergia Mex 2011; 58: 155-61.

20.- SIMMONS M, HETRICK S, JORM A. Shared decisionmaking: benefits, barriers and current opportunities for application. Australas Psychiatry 2010; 18: 394-7.

Correspondencia a:

Prof. Daniel Ciudad Antognini. Klgo., PhD.

Escuela de Kinesiología, Universidad de Valparaíso

Angamos 655. Reñaca, Viña del Mar, Chile.

Email: daniel.ciudad@uv.cl 